НАСЛЕДСТВЕННЫЕ ФОРМЫ ПЕРВИЧНОГО ГИПЕРПАРАТИРЕОЗА

(․ Е.О. Мамедова, Н.Г. Мокрышева, Л.Я. Рожинская

ФГБУ «Национальный медицинский исследовательский центр эндокринологии» Минздрава России, Москва, Россия

Первичный гиперпаратиреоз (ПГПТ) в подавляющем большинстве случаев является спорадическим заболеванием. Наследственные формы ПГПт встречаются значительно реже, однако представляют особый интерес, поскольку позволяют глубже понять патогенез развития новообразований околощитовидных желез в целом. К наследственным формам ПГПТ относят: синдром множественных эндокринных неоплазий 1 типа (МЭН-1), 2А типа (МЭН-2А), 4 типа (МЭН-4), синдром гиперпаратиреоза с опухолью челюсти (hyperparathyroidism-jaw tumour syndrome, HРT-JT), варианты семейной гипокальциурической гиперкальциемии (familial hypocalciuric hypercalcemia, $\mathrm{FHH}$ ) и семейный изолированный гиперпаратиреоз (familial isolated hyperparathyroidism, FIHP). Мутации в генах MEN1, RET, CDKN1B, CDC73 приводят к развитию МЭН-1, МЭН-2А, МЭН-4, НРТ-JТ, соответственно. Варианты FНН обусловлены мутациями в генах CASR, AP2S1, GNA11. Ген(ы), ответственные за развитие большинства случаев FIHP, до сих пор неизвестны.

Выявление наследственной природы ПГПТ важно как для пациента, так и для его родственников первой линии родства, поскольку позволяет определить необходимость скрининга для выявления других компонентов синдромов, в ряде случаев определяет объем операции по поводу ПГПТ, а также риск развития заболевания у потомков.

В статье представлены данные об особенностях наследственных синдромов, в рамках которых возникает ПГПТ, а также характерные особенности ПГПТ в рамках этих синдромов.

КЛЮЧЕВЫЕ СЛОВА: Первичный гиперпаратиреоз; синдром множественных эндокринных неоплазий 1 типа; синдром гиперпаратиреоза с опухолью челюсти; семейная гипокальциурическая гиперкальциемия; семейный изолированный гиперпаратиреоз

\title{
HEREDITARY FORMS OF PRIMARY HYPERPARATHYROIDISM
}

(c) Elizaveta O. Mamedova, Natalya G. Mokrysheva, Liudmila Ya. Rozhinskaya

Endocrinology Research Centre, Moscow, Russia

Primary hyperparathyroidism (PHPT) is sporadic in the majority of cases. Hereditary forms of PHPT are rarer, however, they are of particular interest because they allow a deeper understanding of pathogenesis of parathyroid neoplasia. Hereditary forms of PHPT include multiple endocrine neoplasia type 1 (MEN-1), type 2A (MEN-2A), type 4 (MEN-4), hyperparathyroidism-jaw tumour syndrome (HPT-JT), variants of familial hypocalciuric hypercalcemia (FHH) and familial isolated hyperparathyroidism (FIHP). Mutations in the following genes cause the development of MEN-1, MEN-2A, MEN4, HPT-JT:MEN1, RET, CDKN1B, CDC73, respectively. Variants of FHH are caused by mutations in CASR, AP2S1, GNA11. Gene(s) responsible for the development of the majority of FIHP cases remain unknown.

Identification of hereditary forms of PHPT is important for patients and their first-degree relatives, as it allows defining the necessity of screening to reveal other components of the syndrome, in some cases determines the surgical approach to PHPT, and the risk of development of the disease in offsprings.

This article provides information on hereditary syndromes associated with PHPT and special features of PHPT in each syndrome.

KEYWORDS: Primary hyperparathyroidism; Multiple endocrine neoplasia type 1; Hyperparathyroidism-jaw tumor syndrome; Familial hypocalciuric hypercalcemia; Familial isolated hyperparathyroidism.

\section{ВВЕДЕНИЕ}

В последние десятилетия сформировалось классическое представление о первичном гиперпаратиреозе (ПГПТ) как о заболевании, которое чаще выявляется у женщин в постменопаузе, в большинстве случаев в виде мягких форм. Как правило, заболевание обусловлено солитарной аденомой околощитовидной железы (ОЩЖ) и приблизительно в 90-95\% случаев является спорадическим [1]. Тем не менее, в 1 из 20 случаев ПГПТ может возникать как компонент одного из наследственных синдромов, к которым относятся: синдром множественных эндокринных неоплазий 1 типа (МЭН-1), 2А типа (МЭН-2А), 4 типа (МЭН-4), синдром гиперпаратиреоза с опухолью челюсти (hyperparathyroidism-jaw tumour syndrome, HРT-JT), семейная гипокальциурическая гиперкальциемия (familial hypocalciuric hypercalcemia, $\mathrm{FHH}$ ) и семейный изолированный гиперпаратиреоз (familial isolated hyperparathyroidism, FIHP) [2].

Установление или исключение наследственной природы ПГПТ важно для пациента и его родственников первой линии родства. В случае подтверждения наследственной природы ПГПТ существует вероятность рецидива заболевания после оперативного лечения. При этом необходим регулярный пожизненный скрининг с целью раннего выявления и лечения других компонентов наследственных синдромов, а также молекулярно-генетическое обследование родственников первой линии род- 
ства для выявления бессимптомных носителей мутаций. В случае исключения наследственной природы ПГПТ возможно прогнозировать отсутствие рецидива ПГПт после оперативного лечения в дальнейшем. Также исключается риск развития соответствующих компонентов наследственных синдромов, необходимость обследования родственников первой линии родства и риск развития заболевания у потомков [2].

Каждый наследственный синдром, в рамках которых может возникать ПГПТ, имеет свои характерные клинические особенности, которые будут рассмотрены далее в нашей статье.

\section{СИНДРОМЫ МНОЖЕСТВЕННЫХ ЭНДОКРИННЫХ НЕОПЛАЗИЙ}

Синдром множественных эндокринных неоплазий 1 типа (МЭН-1)

Синдром МЭН-1 (ОМІМ \#131100) был впервые описан в 1954 году, и характеризуется сочетанным развитием опухолей ОЩж, аденогипофиза и островков поджелудочной железы (Пж) [2, 3]. Также в рамках синдрома встречаются опухоли надпочечников, нейроэндокринные опухоли (НЭО) тимуса, легких и желудочно-кишечного тракта. Неэндокринные проявления МЭН-1 включают ангиофибромы, коллагеномы, липомы, лейомиомы, менингиомы и другие опухоли [3]. Недавно рак молочной железы был описан как одно из проявлений синдрома [4]. В соответствии с международными клиническими рекомендациями от 2012 г, клинический диагноз синдрома МЭН-1 основывается на сочетании минимум двух из трех основных МЭН-1-ассоциированных эндокринных опухолей [3]. Распространенность МЭН-1 составляет 2-3 на 100000 человек с одинаковым поражением и женщин, и мужчин [3]. МЭН-1 - это заболевание с аутосомно-доминантным типом наследования, обусловленное герминальными мутациями в гене MEN1. Ген MEN1 был идентифицирован в 1997 г. и расположен на длинном плече хромосомы 11 (11q13) [5]. MEN1 является геном-супрессором опухолевого роста и кодирует менин - белок, состоящий из 610 аминокислотных остатков и преимущественно локализующийся в ядре. Функции менина до конца малоизучены [6].

Некоторые клинические особенности ПГПт в рамках МЭН-1, отличающие его от спорадического ПГПТ, хорошо известны [7]. ПГПТ развивается у 75-95\% пациентов с МЭН-1 и обычно является первым проявлением синдрома (около 75\%) [7, 8, 9]. В целом, частота МЭН-1 при ПГПТ оценивается как 1-18\% [3, 7]. Дебют ПГПТ в рамках МЭН-1 обычно приходится на возраст 20-25 лет, в отличие от спорадического ПГПТ, при котором типичный возраст дебюта - 50-55 лет [3, 7, 10]. Также отмечается одинаковое поражение женщин и мужчин (1:1 vs 3:1 при спорадическом ПГПТ) [3,7]. Клинические проявления различны: у пациентов может быть как асимптомная гиперкальциемия, так и нефролитиаз, фиброзно-кистозная остеодистрофия, симптомы, ассоциированные с гиперкальциемией (полиурия, полидипсия), или пептические язвы. Обычно гиперкальциемия выражена умеренно, а тяжелая гиперкальциемия, приводящая к гиперкальциемическому кризу встречаются редко [3]. В отличие от спорадического ПГПТ, обусловленного в большинстве случаев солитарной аденомой ОЩЖ, ПГПТ при МЭН-1 вызван множественным поражением (гиперплазией, аденомами) ОЩж [3, 7]. Дооперационные визуализирующие методики (УЗИ и сцинтиграфия с технетрилом) имеют ограниченные возможности в связи с потенциальным вовлечением всех ОЩЖ в патологический процесс. Рекомендации по объему хирургического лечения противоречивы: следует ли выполнять субтотальную или тотальную паратиреоидэктомию (ПТЭ) и когда выполнять оперативное вмешательство (на ранних или более поздних стадиях заболевания). Рекомендуется проводить двустороннюю ревизию шеи (в противоположность минимально инвазивной ПТЭ при спорадическом ПГПТ), поскольку в большинстве случаев поражены все четыре ОЩж (множественные аденомы или гиперплазия), хотя в некоторых случаях дифференцировать их гистологически может быть сложно [3, 7]. Необходимо отметить, что злокачественное поражение ОЩЖ крайне редко, но может встречаться у пациентов с синдромом МЭН-1: в литературе описаны единичные случаи (около 12) рака ОЩЖ в рамках МЭН-1 $[11,12,13]$. При ПГПТ в рамках МЭН1 чаще развивается рецидив после оперативного вмешательства по сравнению со спорадическим ПГПТ [7, 8, 9].

В единственной найденной нами работе, посвященной изучению особенностей течения синдрома МЭН-1 у пациентов в возрасте до 21 года, из 160 молодых пациентов с МЭН-1 ПГПт был первым проявлением у 90 пациентов (56\%), и в большинстве случаев был выявлен при биохимическом скрининге (71/90, 79\%) [14]. В целом ПГПТ был выявлен у 122 (76,25\%), из них у 110 (90\%) в возрасте старше 10 лет. У 21 пациента (17\%) наблюдались клинические симптомы (18 (86\%) - нефролитиаз, 7 (33\%) утомляемость, 2 (1\%) боли в костях), при этом самый ранний возраст манифестации был 8 лет (нефролитиаз). Только у 3 пациентов ПГПт возник в возрасте до 6 лет и имел асимптомное течение [14].

В исследовании C. Eller-Vainicher и коллег определялись клинические и биохимические признаки ПГПт, по которым можно заподозрить синдром МЭН-1, а также сравнивалась тяжесть ПГПТ в рамках МЭН-1 и спорадического ПГПТ [7]. При сравнении 469 пациентов со спорадическим ПГПТ и 64 пациентов с ПГПТ в рамках МЭН-1 было показано, что у пациентов с МЭН-1 были более низкие показатели Z-критерия в позвонках и шейке бедра, а также более низкие показатели уровня сывороточного фосфора и паратгормона (ПТГ). При исследовании только пробандов, МЭН-1 был ассоциирован с показателями уровня ПТГ в пределах референсных значений и более молодым возрастом. Сочетание показателей ПТГ в пределах нормальных значений с возрастом менее 50 лет имело сильную ассоциацию с наличием синдрома МЭН-1. В заключении авторы делают вывод, что пациенты с ПгПТ в рамках МЭН-1 имеют более тяжелое поражение костей, но одинаковое поражение почек, несмотря на более мягкие биохимические показатели в сравнении с пациентами со спорадическим ПГПТ [7].

В работе D.M. Lourenço Jr и коллег была обследована группа из 36 пациентов с ПгПт из 8 неродственных семей с МЭН-1 [9]. При этом пациенты были разделены на две группы в соответствии с возрастом - моложе 50 лет (21 человек) и старше 50 лет (15 человек). Было показано, что поражения костей и почек (нефролитиаз) 
при МЭН-1-ассоциированном ПГПТ возникают раньше, чаще, являются более выраженными и прогрессирующими, по сравнению со спорадическим ПГПТ. Уровни ПТГ и кальция крови при МЭН-1-ассоциированном ПГПт были умеренно повышены, несмотря на, в целом, большой процент выявленной деминерализации костей (77,8\%). В подгруппе молодых пациентов (<50 лет) чаще встречалась деминерализация в дистальной 1/3 лучевой кости, и она была более выраженной. В группе пациентов старше 50 лет была выше частота деминерализации костей во всех зонах. Нефролитиаз возникал раньше (<30 лет), чаще (75\%) и был ассоциирован с сопутствующими почечными осложнениями (50\%) и почечной недостаточностью в группе пациентов старшего возраста (33\%). Примечательно, что на момент диагностики ПГПТ 27/36 (75\%) имели манифестную форму заболевания (нефролитиаз). Девять из 36 (9\%) пациентов не имели симптомов, однако у 2 развился нефролитиаз спустя 6 и 36 месяцев после диагностики ПГПТ, соответственно. 8 из 9 асимптомных пациентов были моложе 30 лет, и все 9 были статистически достоверно моложе симптомных пациентов. Авторы делают вывод, что асимптомная фаза при МЭН-1-ассоциированном ПГПТ длится относительно недолго, поскольку 60\% пациентов моложе 30 лет имели симптомы ПГПТ (нефролитиаз). Механизмы, лежащие в основе большей предрасположенности к нефролитиазу при МЭН-1-ассоциированном ПГПТ по сравнению со спорадическим ПГПТ остаются неясными [9].

По данным пилотного эпидемиологического исследования в России, распространенность синдрома МЭН-1 среди пациентов с ПГПТ составила 6,6\%, и была значительно больше среди пациентов с рецидивом ПГПТ $(21,6 \%)[15]$. Течение ПГПТ и нарушения фосфорно-кальциевого обмена при МЭН-1 не зависят от того, являлся ли ПГПТ первым компонентом МЭН-1 или был выявлен при скрининге среди пациентов с опухолями эндокринных желез. У пациентов с МЭН-1 в сравнении с пациентами со спорадическим ПГПТ значимо меньше возраст дебюта заболевания и уровень ПТГ, чаще свойственно множественное поражение ОЩж и малосимптомное течение ПГПТ, особенно в возрасте до 30 лет. Пациенты с возрастом дебюта ПГПт до 40 лет в сочетании с умеренным повышением уровня ПТГ (не более 2,5 раз выше верхней границы референсного диапазона) имеют большую вероятность наличия у них ПГПТ в рамках МЭН-1 (в 12,5 раз) вне зависимости от пола и клинических проявлений ПГПТ (специфичность 97\%, прогностическая ценность - 66,7\%) [15].

В международных рекомендациях по синдрому МЭН1 от 2012 г. приведены следующие показания к анализу гена MEN1: пациент с двумя и более основными МЭН-1-ассоциированными опухолями (опухоли ОЩЖ, аденомы гипофиза, НЭО ПЖ); родственники первой линии родства известных носителей мутаций в гене MEN1; у пациентов, подозрительных на наличие синдрома МЭН-1 (например, у лиц с опухолями ОЩЖ, возникшими до 30 лет; одновременное поражение нескольких ОЩЖ, гастриномы или множественные НЭО ПЖ независимо от возраста; у пациентов с двумя и более МЭН-1-ассоциированными опухолями, но не из классической триады, например при сочетании ПГПТ и опухоли надпочечника) [3].
Синдром множественных эндокринных неоплазий 2А типа (МЭН-2А)

Синдром МЭН-2А (ОМІМ\#171400) является клиническим вариантом синдрома МЭН-2, проявляется медуллярным раком щитовидной железы (100\%), феохромоцитомой (50\%), ПГПТ (20-30\%), и обусловлен герминальными мутациями в гене RET [2]. Примечательно, что при синдроме МЭН-2А в подавляющем большинстве случаев ПГПТ не бывает первым проявлением синдрома, а выявляется либо во время операции по поводу медуллярного рака щитовидной железы, либо спустя годы после операции по поводу медуллярного рака. Средний возраст на момент диагностики составляет 38 лет $[2,16]$. Нами было найдено описание единичного клинического случая девочки 5 лет из семьи с МЭН-2А, у которой перед проведением профилактической тиреоидэктомии (в отсутствие патологии щитовидной железы) был диагностирован ПГПТ [17]. Обычно ПГПТ при МЭН-2А обусловлен солитарной аденомой ОЩЖ, хотя может встречаться и гиперплазия нескольких ОЩЖ [16].

\section{Синдром множественных эндокринных неоплазий}

4 типа (МЭН-4)

Синдром МЭН-4 (ОМІМ \#610755) был впервые описан в 2002 г. A. Fritz и коллегами как «МЭН-подобное» аутосомно-рецессивное заболевание у крыс [18]. В течение первого года жизни у животных развивались двусторонние феохромоцитомы, множественные параганглиомы, медуллярный рак щитовидной железы, двусторонняя гиперплазия ОЩЖ, аденомы гипофиза, при отсутствии мутаций в генах RET и MEN1 [18]. В 2006 г. N. Pellegata с коллегами выявили мутацию в гене $C D K N 1 B$, кодирующем ингибитор клеточного цикла р27, у крыс с «МЭН-подобным» фенотипом (развитие опухолей, характерных как для МЭН-1, так и для МЭН-2) [19]. Первым доказанным случаем заболевания у человека, которое в последующем было названо МЭН-4, стала семья из Германии с семейными аденомами гипофиза, ПГПТ, ангиомиолипомой почки и раком яичек среди различных членов семьи. Генетическое исследование показало наличие нонсенс-мутации в гене CDKN1B в отсутствие мутаций в гене MEN1 [19]. Вскоре после этого, герминальная гетерозиготная мутация со сдвигом рамки считывания в гене CDKN1B была описана в другом «МЭН-1-подобном» случае, негативном по мутации в гене MEN1: женщина из Голландии с мелкоклеточной карциномой шейки матки, АКТГ-продуцирующей аденомой гипофиза и гиперпаратиреозом [20]. Ген CDKN1B расположен на коротком плече 12 хромосомы (12p13) и кодирует повсеместно экспрессирующийся белок р27 (Кір1), состоящий из 198 аминокислотных остатков. Он принадлежит, совместно с р21 и р57, к семейству циклин-зависимых ингибиторов киназ (KIP/CIP), регулирующих переход клеточного цикла из фазы G1 в S фазу. Менин и RET также являются компонентами р27-зависимого пути, который регулирует тканеспецифическую клеточную пролиферацию. К 2013 г было описано 12 случаев герминальных мутаций в гене CDKN1B [21], в дальнейшем было описано еще несколько случаев $[22,23]$. С учетом небольшого количества выявленных пациентов, описать фенотип МЭН-4 сложно. Возраст манифестации клинических проявлений обычно на 20 лет позже, чем при МЭН-1 
(36-79 лет), чаще около 50-60 лет. ПГПТ имеет высокую пенетрантность (81\%) и часто бывает первым клиническим проявлением. В большинстве случаев поражаются несколько ОЩЖ. Аденомы гипофиза являются вторым по частоте клиническим проявлением (46\%) [21]. Другие опухоли включают карциноиды бронхов, энтеропанкреатические НЭО, рак яичек, опухоли надпочечников, ангиомиолипомы почек [24]. Диагноз МЭН-4 может быть заподозрен у пациентов с фенотипом МЭН-1 в отсутствие мутаций в гене MEN1 (фенокопий МЭН-1).

\section{СИНДРОМ ГИПЕРПАРАТИРЕОЗА С ОПУХОЛЬЮ ЧЕЛЮСТИ}

Синдром гиперпаратиреоза с опухолью челюсти (hyperparathyroidism-jaw tumour syndrome, HРT-JT) (OMIM \#145001) является редким заболеванием с аутосомно-доминантным типом наследования [25]. Синдром характеризуется развитием ПГПТ, оссифицирующих фибром нижней и/или верхней челюсти, опухолями матки и, реже, поражением почек [24, 26]. Истинная распространенность синдрома НРТ-JТ до сих пор неизвестна. К 2015 г было описано менее 300 случаев заболевания из приблизительно 100 семей [24]. Несмотря на название синдрома, опухоли челюсти встречаются лишь в 30-40\% случаев [26]. Оссифицирующие фибромы являются доброкачественными, но могут нарушать нормальное расположение зубов и приводить к косметическим дефектам. Опухоли иногда могут быть двусторонними/ мультифокальными и могут рецидивировать или продолжать расти при отсутствии хирургического лечения. Указанные опухоли следует дифференцировать от бурых опухолей при агрессивно протекающем ПГПТ, рост которых останавливается и обычно наблюдается их регрессия после наступления ремиссии ПГПТ $[27,28]$. Опухоли матки (лейомиомы, гиперплазия эндометрия, аденосаркомы, аденофибромы, множественные аденоматозные полипы) встречаются у 57,3\% больных женщин [24]. Поражение почек (гамартомы, поликистоз почек, опухоли Вильмса, аденокарциномы) встречается в 13,3\% случаев HPT-JT [24]. Также сообщалось о выявлении аденокарциномы ПЖ, смешанных герминогенных опухолей яичка, рака щитовидной железы и толстой кишки, но данные о том, что их частота выше при НРТ-JТ по сравнению с общей популяцией, отсутствуют [26].

НРТ-JТ обусловлен герминальными мутациями в гене-супрессоре опухолевого роста CDC73 (cell division cycle protein 73 homolog), расположенном на длинном плече 1 хромосомы (1q31.2), идентифицированном в 2002 г. и ранее обозначавшимся как HRPT2 [29]. Ген кодирует белок парафибромин, состоящий из 531 аминокислотного остатка [29]. Парафибромин является одной из пяти субъединиц ассоциированного с полимеразой комплекca 1 (polymerase-associated complex 1, PAF1) - ключевого регулирующего транскрипцию комплекса, который взаимодействует непосредственно с РНК-полимеразой II [30]. До сих пор не было выявлено корреляций генотип-фенотип для мутаций в CDC73.

ПГПТ является основным проявлением НРТ-ЈТ и выявляется приблизительно в 95\% случаев [26]. Частота ПГПТ увеличивается с возрастом, и хотя обычно ПГПТ манифестирует в раннем молодом возрасте (самый ранний опи- санный возраст дебюта ПГПТ - 7 лет [31], самый ранний описанный возраст при карциноме ОЩЖ - 20 лет [32]), дебют ПГПТ может быть и на шестом десятке жизни [33]. ПгПт может протекать мягко, однако в случае карциномы ОЩЖ течение может быть тяжелым, с гиперкальциемическими кризами. ПГПТ в рамках НРТ-JТ обычно вызван единичной доброкачественной аденомой ОЩж, кистозной или с атипичными гистологическими характеристиками [24]. В отличие от других наследственных вариантов ПГПТ, карцинома ОЩЖ в рамках НРТ-ЈТ встречается в 10-21,6\% случаев [24, 26]. Мультигландулярное поражение встречается редко при первичной операции (20\% случаев), вторая аденома ОЩЖ может возникнуть метахронно, спустя годы или десятилетия после возникновения первичной опухоли (23,9\% случаев) [24].

Оптимальный хирургический подход при ПГПТ в рамках НРТ-JT пока не разработан. Ранее рядом авторов предлагалось проведение двусторонней ревизии шеи и субтотальной ПТЭ или тотальной ПТЭ с или без аутотрансплантации, в связи с риском мультигландулярного поражения и злокачественности [24]. Однако аутотрансплантация может быть ассоциирована с диссеминацией раковых клеток. В последнее время чаще предлагается селективная ПТЭ при поражении одной ОЩЖ и отсутствии подозрения на злокачественность. Такая стратегия обеспечивает меньший риск развития гипопаратиреоза $[24,34]$. В случае подозрения на рак ОЩЖ (большие образования ОЩЖ с инфильтративным ростом, крайне высокие показатели кальция и ПТГ) предпочтительно выполнение резекции единым блоком с удалением ипсилатеральной половины щитовидной железы, прилежащих мягких тканей и ипсилатеральной ОЩЖ с целью предотвращения повреждения опухоли и риска диссеминации [24].

\section{ВАРИАНТЫ СЕМЕЙНОЙ ГИПОКАЛЬЦИУРИЧЕСКОЙ ГИПЕРКАЛЬЦИЕМИИ}

\section{Семейная гипокальциурическая гиперкальциемия}

\section{1, 2, 3 типов}

Семейная гипокальциурическая гиперкальциемия (familial hypocalciuric hypercalcemia, FHH), впервые описанная в 1972 году, является редким заболеванием с аутосомно-доминантным типом наследования [35]. Диагноз FHH можно заподозрить при сочетании гиперкальциемии и нормального или несколько повышенного уровня ПТГ и относительной гипокальциурии. Концепция относительной гипокальциурии при $\mathrm{FHH}$ заключается в сниженной экскреции кальция с мочой при сочетании с высоким уровнем кальция крови, что свидетельствует об отсутствии гиперкальциурического ответа на гиперкальциемию [2]. Поскольку экскреция кальция с мочой за определённый интервал времени значительно зависит от скорости клубочковой фильтрации (СКФ) и продолжительности времени сбора мочи, общая экскреция кальция не является ценным показателем для дифференциальной диагностики случаев $\mathrm{FHH}$ от типичного ПГПТ. Поэтому с целью дифференциальной диагностики ПГПТ и $\mathrm{FHH}$ используется расчет отношения почечного клиренса кальция к клиренсу креатинина (UCCR), который при FHН составляет менее 0,01 (формула для расче- 
та $\mathrm{CaCl} / \mathrm{CrCl}=[\mathrm{Cau} \times \mathrm{Crs}] /[\mathrm{Cru} \times \mathrm{Cas}]$, где $\mathrm{CaCl}$ - клиренс кальция, $\mathrm{CrCl}$ - клиренс креатинина, $\mathrm{Cau}$ - концентрация кальция в моче (ммоль/л), Crs - концентрация креатинина в сыворотке крови (мкмоль/л), Cru - концентрация креатинина в моче (мкмоль/л), Cas - концентрация кальция в сыворотке крови (ммоль/л)) [2, 24]. Для ПГПТ индекс UCCR обычно составляет более 0,02 , однако в исследовании S.E. Christensen и коллег было продемонстрировано, что отрезная точка UCCR менее 0,01 без исследования гена CASR выявит только 65\% пациентов с FHН и неверно классифицирует 4\% пациентов с ПГПТ как имеющих $\mathrm{FHH}$. Показатель UCCR в пределах 0,01-0,02 имеют 33\% пациентов с ПГПТ, и 35\% с FHH имеют UCCR $\geq 0,01$ [36].

У пациентов с FHН симптоматика обычно слабо выражена или отсутствует, и гиперкальциемия чаще выявляется случайно. Гораздо реже, чем при классическом ПГПТ, могут встречаться быстрая утомляемость, слабость и полидипсия, нефролитиаз. На рентгенограммах костей и рентгеновской денситометрии патологии обычно не выявляют. При FHН могут встречаться хондрокальциноз и преждевременная кальцификация сосудов, обычно не проявляющие себя клинически. Гиперкальциемия имеет 100\% пенетрантность во всех возрастных группах $[2,24]$. Субтотальная ПТЭ при FHН приводит лишь к транзиторному снижению уровня кальция сыворотки крови, с последующей персистенцией гиперкальциемии спустя несколько дней после операции, поскольку ПТЭ не позволяет скорректировать патологию кальций-зависимого порога секреции ПТГ. Из-за обычно доброкачественного течения заболевания и отсутствия ответа на субтотальную ПТЭ, только небольшое количество пациентов следует подвергать ПтЭ. В редких случаях, таких как рецидивирующий панкреатит, очень высокий уровень ПТГ, или очень высокий уровень кальция (постоянно выше 3,5 ммоль/л) может быть показана субтотальная ПТЭ или даже тотальная ПТЭ [2].

В настоящее время выделяют три типа $\mathrm{FHH}$, обусловленных, соответственно: инактивирующими мутациями в гене кальций-чувствительного рецептора (CASR) (3q21.1) - FHH 1 типа (OMIM \#145980), мутациями в гене GNA11 (19p13.3), кодирующем Ga11 - FHH 2 типа (OMIM \#145981) [37], мутациями в гене AP2S1 (19q13.2-q13.3), кодирующем б-2 субъединицу адаптор-связанного белкового комплекса 2 (adaptor-related protein complex 2, б-2 subunit) - FHH 3 типа (OMIM \#600740) [38]. Большинство случаев FHH обусловлено гетерозиготными инактивирующими мутациями в гене CASR. Кроме того, инактивирующие гомозиготные мутации в CASR приводят также к развитию неонатального тяжелого гиперпаратиреоза и аутосомно-доминантного мягкого гиперпаратиреоза [2]. Около 30\% пробандов и семей с FHH не имеют мутаций в гене CASR, при этом у части из них выявляются мутации в генах AP2S1 и GNA11, но у некоторых мутации не обнаруживаются [39].

Неонатальный тяжелый гиперпаратиреоз

(neonatal severe hyperparathyroidism, NSHPT)

NSHPT, описанный как единичный случай в 1947 г. и у двух членов одной семьи в 1964 г., является чрезвычайно редким заболеванием новорожденных (ОМІМ \#239200) с жизнеугрожающей, тяжелой гиперкальциемией, очень высоким уровнем ПТГ, переломами ре- бер, гипотонией, респираторным дистресс-синдромом, и значительным увеличением всех ОЩЖ [40]. ПГПТ выявляется при рождении или в течение шести первых месяцев жизни и является причиной тяжелой симптоматической гиперкальциемии, деминерализации костей и задержки в развитии. Ранняя диагностика очень важна, поскольку при отсутствии лечения NSHPT может приводить к тяжелым расстройствам развития нервной системы. NSHPT обусловлен инактивирующими гомозиготными или компаунд-гетерозиготными мутациями в гене CASR [2]. Диагноз основан на клинических проявлениях и/или наличии $\mathrm{FHH}$ у родственников первой линии родства. Методом выбора в лечении является экстренная тотальная ПТЭ [2, 24].

Аутосомно-доминантный мягкий гиперпаратиреоз

(autosomal dominant moderate (mild)

hyperparathyroidism, ADMH)

ADMH (OMIM \#601199) является редким синдромом, описанным T. Carling с коллегами в 2000 году в большой семье из Швеции: у 20 членов семьи были фенотипы FHH и FIHP, гиперкальциемия и гиперкальциурия, несоответственно высокий уровень ПТГ, а так же нефролитиаз у части пациентов. Генетическое исследование данных членов семьи показало атипичную герминальную инактивирующую мутацию в интрацитоплазматическом домене кальций-чувствительного рецептора (F881L) [41]. Методом выбора в лечении является хирургический, поскольку было показано, что после субтотальной или тотальной ПТЭ наблюдалась регрессия гиперкальциемии [42].

\section{СЕМЕЙНЫЙ ИЗОЛИРОВАННЫЙ ГИПЕРПАРАТИРЕОЗ}

FIHP (OMIM \#145000) является редким заболеванием с аутосомно-доминантным типом наследования, характеризующимся развитием опухолей одной или нескольких ОЩЖ и отсутствием других опухолей эндокринных и неэндокринных органов. FIHP диагностируют в семьях с ПГПТ как единственной эндокринопатией, в отсутствие специфических проявлений других синдромов (МЭН-1, HPT-JT, FHH) [2]. К 2013 г. было описано более 100 семей c FIHP, и у большинства из них генетическая причина остается неизвестной $[2,43]$. Иногда FIHP может представлять собой неполный вариант других синдромов (МЭН-1, HPT-JT, FHH). В некоторых семьях с FIHP были выявлены герминальные мутации в генах $\operatorname{MEN} 1$ (20-23\%) [44, 45, 46], CASR (14-18\%) [43, 46] и CDC73 (0-33\%) [26, 46]. Лечение FIHP хирургическое, однако оптимальная тактика не разработана. В случае солитарного поражения ОЩЖ возможно проведение ПТЭ. В случае мультигландулярного поражения рекомендовано проведение субтотальной ПТЭ. При выявлении мутаций в генах MEN1 или CDC73 показано лечение как при соответствующих синдромах [24].

В 2016 г опубликована работа по секвенированию экзома в 8 семьях с FIHP, и в трех из них были выявлены три редких миссенс варианта в гене GCM2, кодирующем транскрипционный фактор, необходимый для развития околощитовидных желез [47]. Авторы предполагают, что GCM2 является протоонкогеном для ОЩЖ, и что активирующие мутации в GCM2 могут приводить к развитию FIHP [47]. 


\section{ЗАКЛЮЧЕНИЕ}

В настоящее время известно шесть наследственных синдромов, в рамках которых возникает ПГПТ. Наследственный ПГПТ обычно возникает в более молодом возрасте по сравнению со спорадическим ПГПТ, встречается одинаково часто у мужчин и женщин. Верификация наследственной природы ПГПТ возможна с помощью молекулярно-генетических исследований, однако в ряде наследственных случаев (например, при FIHP), генетическая причина развития ПГПт остается неизвестной. Точное установление диагноза спорадической или наследственной формы ПГПТ определяет объем хирурги- ческого вмешательства, тактику дальнейшего наблюдения и лечения, и необходимость обследования и лечения родственников первой линии родства.

\section{ДОПОЛНИТЕЛЬНАЯ ИНФОРМАЦИЯ}

Конфликт интересов. Обзор является частью диссертационной работы Мамедовой Е.О. Остальные авторы декларируют отсутствие явных и потенциальных конфликтов интересов, связанных с публикацией настоящей статьи.

Источник финансирования. Поисково-аналитическая работа и подготовка статьи проведены на личные средства авторского коллектива.

\section{СПИСОК ЛИТЕРАТУРЫ | REFERENCES}

1. Дедов И.И., Мельниченко Г.А., Мокрышева Н.Г. и др. Первичный гиперпаратиреоз: клиника, диагностика, дифференциальная диагностика, методы лечения // Проблемы эндокринологии. - 2016. - T. 62. - №6. - C. 40-77. [Dedov II, Melnichenko GA, Mokrysheva NG et al. Primary hyperparathyroidism: the clinical picture, diagnostics, differential diagnostics, and methods of treatment. Problemy endocrinologii. 2016;62(6):40-77. (In Russ).] doi: 10.14341/probl201662640-77

2. Giusti F, Cavalli L, Cavalli T, Brandi ML. Hereditary hyperparathyroidism syndromes. J Clin Densitom. 2013;16(1):69-74. doi: 10.1016/j.jocd.2012.11.003

3. Thakker RV, Newey PJ, Walls GV et al. Clinical practice guidelines for multiple endocrine neoplasia type 1 (MEN1). J Clin Endocrinol Metab. 2012;97(9):2990-3011. doi: 10.1210/jc.2012-1230

4. Dreijerink KM, Goudet P, Burgess JR et al. Breast-cancer predisposition in multiple endocrine neoplasia type 1. N Engl J Med. 2014;371(6):583-584. doi: 10.1056/nejmc1406028

5. Cavaco BM, Santos R, Felix A et al. Identification of de novo germline pathogenic variants in the HRPT2 gene in two apparently sporadic cases with challenging parathyroid tumor diagnoses. Endocr Pathol. 2011;22:44-52. doi: 10.1007/s12022-011-9151-1

6. Tsukada T, Nagamura Y, Okhura N. MEN1 gene and its mutations: basic and clinical implications. Cancer Sci. 2009;100(2):209-215. doi: 10.1111/j.1349-7006.2008.01034.x

7. Eller-Vainicher C, Chiodini I, Battista C et al. Sporadic and MEN1-related primary hyperparathyroidism: differences in clinical expression and severety. J Bone Miner Res. 2009;24:1404-1410. doi: 10.1359/jbmr.090304

8. Lourenço DM Jr, Toledo RA, Mackowiak II et al. Multiple endocrine neoplasia type 1 in Brazil: MEN1 founding mutation, clinical features, and bone mineral density profile. Eur J Endocrinol. 2008;159(3):259-274. doi: 10.1530/eje-08-0153

9. Lourenço DM Jr, Coutinho FL, Toledo RA et al. Early-onset, progressive, frequent, extensive, and severe bone mineral and renal complications in multiple endocrine neoplasia type 1-associated primary hyperparathyroidism. J Bone Miner Res. 2010;25(11):2382-2391. doi: 10.1002/jbmr.125

10. Machens A, Schaaf L, Karges W et al. Age-related penetrance of endocrine tumours in multiple endocrine neoplasia type 1 (MEN1): a multicentre study of 258 gene carriers. Clin Endocrinol (Oxf). 2007;67:613-622. doi: 10.1111/j.1365-2265.2007.02934x

11. Del Pozo C, García-Pascual L, Balsells M et al. Parathyroid carcinoma in multiple endocrine neoplasia type 1. Case report and review of the literature. Hormones (Athens). 2011;10(4):326-331. doi: 10.14310/horm.2002.1325

12. Ospina NS, Sebo TJ, Thompson GB et al. Prevalence of parathyroid carcinoma in 348 patients with multiple endocrine neoplasia type 1 - case report and review of the literature. Clin Endocrinol (Oxf). 2016;84(2):244-249. doi: 10.1111/cen.12714

13. Shih RY, Fackler S, Maturo S et al. Parathyroid carcinoma in multiple endocrine neoplasia type 1 with a classic germline mutation. Endocr Pract. 2009;15(6):567-572. doi: 10.4158/ep09045.crr

14. Goudet P, Dalac A, Le Bras A et al. MEN1 disease occurring before 21 years old. A 160-patient cohort study from the GTE (Groupe d'etude des Tumeurs Endocrines). J Clin Endocrinol Metab. 2014;100(4):15681577. doi: 10.1210/jc.2014-3659
15. Ростомян, Л.Г. Синдром множественных эндокринных неоплазий 1 типа: распространенность среди пациентов с первичным гиперпаратиреозом, клинические и молекулярно-генетические характеристики : - дис. ... канд. мед. наук. - М., 2011. - 206 C. [Rostomyan LG. Sindrom mnozhestvennyh ehndokrinnyh neoplazij 1 tipa: rasprostranennost' sredi pacientov s pervichnym giperparatireozom, klinicheskie i molekulyarno-geneticheskie harakteristiki. [dissertation] Moscow; 2011. (In Russ).]

16. Alevizaki M, Saltiki K. Primary hyperparathyroidism in MEN2 syndromes. Recent Results Cancer Res. 2015;204:179-186. doi: 10.1007/978-3-319-22542-5_8

17. Magalhães PK, Antonini SR, de Paula FJ et al. Primary hyperparathyroidism as the first clinical manifestation of multiple endocrine neoplasia type 2A in a 5-year-old child. Thyroid. 2011;21(5):547-550. doi: $10.1089 /$ thy. 2010.0336

18. Fritz A, Walch A, Piotrowska K et al. Recessive transmission of a multiple endocrine neoplasia syndrome in the rat. Cancer Res. 2002;62:3048-3051.

19. Pellegata NS, Quintanilla-Martinez L, Siggelkow H et al. Germline mutations in p27(Kip1) cause a multiple endocrine neoplasia syndrome in rats and humans. Proc Nat Acad Sci USA. 2006;103(42):15558-15563. doi: 10.1073/pnas.0603877103

20. Georgitsi M, Raitila A, Karhu A et al. Germline CDKN1B/p27Kip1 mutation in multiple endocrine neoplasia. J Clin Endocrinol Metab. 2007:92:3321-3325. doi: 10.1210/jc.2006-2843

21. Lee M, Pellegata NS. Multiple endocrine neoplasia type 4. Front Horm Res. 2013;41:63-78. doi: 10.1159/000345670

22. Elston MS, Meyer-Rochow GY, Dray M et al. Early-onset primary hyperparathyroidism associated with a novel germline mutation in CDKN1B. Case Reports in Endocrinology. 2015;510985:1-4. doi: 10.1155/2015/510985

23. Tonelli F, Giudici F, Giusti F et al. A heterozygous frameshift mutation in exon 1 of CDKN1B gene in a patient affected by MEN4 syndrome. Eur J Endocrinol. 2014;171(2):K7-K17. doi: 10.1530/eje-14-0080

24. lacobone M, Carnaille B, Palazzo FF, Vriens M. Hereditary hyperparathyroidism - a consensus report of the European Society of Endocrine Surgeons (ESES). Langenbecks Arch Surg. 2015;400(8):867-886. doi: 10.1007/s00423-015-1342-7

25. Jackson CE, Norum RA, Boyd SB et al. Hereditary hyperparathyroidism and multiple ossifying jaw fibromas: a clinically and genetically distinct syndrome. Surgery. 1990;108:1006-1012.

26. Jackson MA, Rich TA, Hu MI, Perrier N, Waguespack SG. CDC73-Related Disorders. In: GeneReviews [Internet]. Pago RA, Adam MP, Ardinfer HH, Wallace SE, Amemiya A, Bean LJH., Bird TD, Fong CT, Mefford HC, Smith RJH., Stephens K, editors. Seattle (WA):: University of Washington, 1993-2015. Available from: http://www.ncbi.nlm.nih.gov/books/NBK3789/

27. Ennazk L, El Mghari G, El Ansari N. Jaw tumor in primary hyperparathyroidism is not always a brown tumor. Clin Cases Miner Bone Metab. 2016;13(1):64-66. doi: 10.11138/ccmbm/2016.13.1.064

28. Parfitt J, Harris M, Wright JM, Kalamchi S. Tumor suppressor gene mutation in a patient with a history of hyperparathyroidism-jaw tumor syndrome and healed generalized osteitis fibrosa cystica: a case report and genetic pathophysiology review. J Oral Maxillofac Surg. 2015;73(1):194.e1-194.e9. doi: 10.1016/j.joms.2014.09.008 
29. Carpten JD, Robbins CM, Villablanca A et al. HRPT2, encoding parafibromin, is mutated in hyperparathyroidism-jaw tumor syndrome. Nature Genetics. 2002:32(4):676-680. doi: 10.1038/ng1048

30. Newey PJ, Bowl MR, Thakker RV. Parafibromin - functional insights. J Intern Med. 2009;266:84-98. doi: 10.1111/j.1365-2796.2009.02107.x

31. Pichardo-Lowden AR, Manni A, Saunders BD, Baker MJ. Familial hyperparathyroidism due to a germline pathogenic variant of the CDC73 gene: implications for management and age-appropriate testing of relatives at risk. Endocr Pract. 2011;17:602-609. doi: 10.4158/ep10337.ra

32. Harman CR, van Heerden CA, Farley DR. Sporadic primary hyperparathyroidismin young patients: a separate disease entity? Arch Surg. 1999;134(6):651-655. doi: 10.1001/archsurg.134.6.651

33. Bradley KJ, Cavaco BM, Bowl MR et al. Parafibromin pathogenic variants in hereditary hyperparathyroidism syndromes and parathyroid tumours. Clin Endocrinol (Oxf). 2006;64:299-306. doi: 10.1111/j.1365-2265.2006.02460.x

34. lacobone M, Masi G, Barzon L et al. Hyperparathyroidism jaw-tumor syndrome: a report of three large kindred. Langenbecks Arch Surg. 2009;394:817-825. doi: 10.1007/s00423-009-0511-y

35. Foley TP Jr, Harrison HC, Arnaud CD, Harrison HE. Familial benign hypercalcemia. J Pediatr. 1972;81:1060-1067

36. Christensen SE, Nissen PH, Vestergaard P. Discriminative power of three indices of renal calcium excretion for the distinction between familial hypocalciuric hypercalcaemia and primary hyperparathyroidism: a follow-up study on methods. Clin Endocrinol (Oxf). 2008;69:713-720. doi: 10.1111/j.1365-2265.2008.03259.x

37. Nesbit MA, Hannan FM, Howles S et al. Mutations affecting G-protein subunit a11 in hypercalcemia and hypocalcemia. N Engl J Med. 2013;368(26):2476-2486. doi: 10.1056/nejmoa1300253

38. Nesbit MA, Hannan F, Howles SA et al. Mutations in AP2S cause familial hypocalciuric hypercalcemia type 3. Nat Genet. 2012;45(1):93-97. doi: 10.1038/ng.2492
39. Vargas-Poussou R, Mansour-Hendili L, Baron S et al. Familial hypocalciuric hypercalcemia types 1 and 3 and primary hyperparathyroidism: similarities and differences. J Clin Endocrinol Metab. 2016;101(5):2185-2195. doi: 10.1210/jc.2015-3442

40. George J, Acharya SV, Bandgar TR et al. Primary hyperparathyroidism in children and adolescents. Indian J Pediatr. 2010;77:175-178. doi: 10.1007/s12098-009-0289-5

41. Carling T, Szabo E, Bai M et al. Familial hypercalcemia and hypercalciuria caused by a novel mutation in the cytoplasmic tail of the calcium receptor. J Clin Endocrinol Metab. 2000;85(5):2042-2047. doi: 10.1210/jcem.85.5.6477

42. Carling T, Udelsman R. Parathyroid surgery in familial hyperparathyroid disorders. J Intern Med. 2005;257:27-37. doi: 10.1111/j.1365-2796.2004.01428.x

43. Simonds WF, James-Newton LA, Agarwal SK et al. Familial isolated hyperparathyroidism: clinical and genetic characteristics of 36 kindreds. Medicine (Baltimore). 2002;81(1):1-26. doi: 10.1097/00005792-200201000-00001

44. Hannan FM, Nesbit MA, Christie P.T et al. Familial isolated primary hyperparathyroidism caused by mutations of the MEN1 gene. Nat Clin Pract Endocrinol Metab. 2008;4(1):53-58. doi: 10.1038/ncpendmet0718

45. Miedlich S, Lohmann T, Schneyer U et al. Familial isolated primary hyperparathyroidismda multiple endocrine neoplasia type 1 variant? Eur J Endocrinol. 2001;145(2):155-160. doi: 10.1530/eje.0.1450155

46. Warner J, Epstein M, Sweet A et al. Genetic testing in familial isolated hyperparathyroidism: unexpected results and their implications. J Med Genet. 2004;41:155-160. doi: 10.1136/jmg.2003.016725

47. Guan B, Welch JM, Sapp JC et al. GCM2-activating mutations in familial isolated hyperparathyroidism. Am J Hum Genet. 2016;99(5):1034-1044. doi: 10.1016/j.ajhg.2016.08.018. Epub 2016 Oct 13.

\section{ИНФОРМАЦИЯ ОБ АВТОРАХ [AUTHORS INFO]}

* Мамедова Елизавета Октаевна, к.м.H. [Elizaveta O. Mamedova, MD, PhD]; адрес: (почтовый рабочий адрес с индексом) Россия, 117036 Москва, ул. Дмитрия Ульянова, д.11 [address: 11 Dm.Ulyanova street, 117036 Moscow, Russia]; ORCID: http://orcid.org/0000-0002-9783-3599; eLibrary SPIN: 3904-6017; e-mail: lilybet@mail.ru

Мокрышева Наталья Георгиевна, д.м.н. [Natalya G. Mokrysheva, MD, PhD], Россия, 117036, Москва, ул. Дмитрия Ульянова, д.11 [address: 11 Dm.Ulyanova street, 117036 Moscow, Russia]; ORCID: http://orcid.org/0000-0002-9717-9742; eLibrary SPIN: 5624-3875; e-mail: nm70@mail.ru

Рожинская Людмила Яковлевна, д.м.н., професcop [Liudmila Ya. Rozhinskaya, MD, PhD, Professor]; ORCID: http:// orcid.org/0000-0001-7041-0732; eLibrary SPIN: 5691-7775; e-mail: Irozhinskaya@gmail.com.

\section{ЦИТИРОВАТЬ:}

Мамедова Е.О., Мокрышева Н.Г., Рожинская Л.Я. Наследственные формы первичного гиперпаратиреоза. // Остеопороз и остеопатии. — 2018. — Т. 21. — №2. — С.23-29. doi: 10.14341/osteo9877

\section{TO CITE THIS ARTICLE:}

Mamedova EO, Mokrysheva NG, Rozhinskaya LY. Hereditary forms of primary hyperparathyroidism. Osteoporosis and bone diseases. 2018;21(2):23-29 doi: 10.14341/osteo9877 